\title{
Effect of mouth leak on effectiveness of nasal bilevel ventilatory assistance and sleep architecture
}

\author{
H. Teschler*, J. Stampa*, R. Ragette*, N. Konietzko*, M. Berthon-Jones**
}

\begin{abstract}
Effect of mouth leak on effectiveness of nasal bilevel ventilatory assistance and sleep architecture. H. Teschler, J. Stampa, R. Ragette, N. Konietzko, M. Berthon-Jones. (C)ERS Journals Ltd 1999.

ABSTRACT: Mouth leak is common during nasal ventilatory assistance, but its effects on ventilatory support and on sleep architecture are unknown. The acute effect of sealing the mouth on sleep architecture and transcutaneous carbon dioxide tension $\left(\mathrm{Ptc}, \mathrm{CO}_{2}\right)$ was tested in 9 patients ( 7 hypercapnic) on longterm nasal bilevel ventilation with symptomatic mouth leak.

Patients slept with nasal bilevel ventilation at their usual settings on two nights in random order. On one night, the mouth was taped closed. Leak was measured with a pneumotachograph.

Median leak fell from $0.35 \pm 0.07$ (mean \pm SEM) $L \cdot \mathrm{s}^{-1}$ untaped to $0.06 \pm 0.03 \mathrm{~L} \cdot \mathrm{s}^{-1}$ taped. $\mathrm{Ptc}_{\mathrm{CO}} \mathrm{CO}_{2}$ fell in 8/9, including all hypercapnic patients. Across all patients, the mean $P$ tc, $\mathrm{CO}_{2}$ fell by $1.02 \pm 0.28 \mathrm{kPa}(7.7 \pm 2.1 \mathrm{mmHg})$ with taping $(\mathrm{p}=0.007)$. Arousal index fell in every patient. Mean arousal index fell from $35.0 \pm 3.0$ to $13.9 \pm 1.2 \cdot h^{-1}(p<0.0001)$, and rapid eye movement (REM) sleep increased from $12.9 \pm 1.5 \%$ to $21.1 \pm 1.8 \%$ sleep time $(p=0.0016)$. Slow wave sleep changed inconsistently, from a mean of $13.1 \pm 1.6 \%$ to $19.5 \pm 2.2 \%$ of sleep $(p=0.09)$. Sleep latency and efficiency were unchanged. In four healthy volunteers ventilator-induced awake hypopharyngeal pressure swing during timed bilevel ventilation fell by $35 \pm 5 \% \cdot \mathrm{L}^{-1} \cdot \mathrm{s}^{-1}$ of voluntary mouth leak $(\mathrm{p}<0.0001)$.

Mouth leak reduces effective nasal bilevel ventilatory support, increases transcutaneous carbon dioxide tension, and disrupts sleep architecture.

Eur Respir J 1999; 14: 1251-1257.
\end{abstract}

Nocturnal nasal bilevel ventilatory support has become an important and successful therapy for respiratory failure during sleep [1-3]. At present, nasal masks are more common than mouthpieces, mouth-nose masks or full face masks (perhaps because of issues related to comfort, fit and deadspace) but a major practical problem is escape of air via the mouth, as has been well documented for nasal continuous positive airway pressure (CPAP) [4, 5].

In the only study to date to directly measure the effect of mouth leaks on effectiveness of ventilatory support, CARREY et al. [6] investigated the effect of voluntary mouth opening on diaphragm electromyogram (EMG) activity during awake nasal intermittent positive pressure ventilation in 5 subjects. With the mouth closed, diaphragm EMG dropped to $15 \%$ of unassisted control, but with the mouth open, returned to $98 \%$ of unassisted control, implying complete loss of ventilatory support.

Mouth leak is ubiquitous during noninvasive ventilatory support in sleep. BACH et al. [7] demonstrated that during nasal intermittent positive pressure ventilation (NIPPV), severe leak ( $>33 \%$ of tidal volume escaping) was present for a median of $55 \%$ of sleep time, and was associated with 7.5 desaturations (of at least 4\%) per hour of sleep.

For editorial comments see page 1241
*Ruhrlandklinic, Faculty of Medicine,
University of Essen, Germany. **ResUniversity of Essen, German
Med Ltd, Sydney, Australia.

Correspondence: H. Teschler

Ruhrlandklinik

Tüschener Weg 40

D-45239 Essen

Germany

Fax: 492014334049

Keywords: Arousal

bilevel ventilation

hypopharyngeal pressure

mouth leak

sleep

Received: January 311999

Accepted after revision August 141999
MEyER et al. [8] found severe mouth leak during most of sleep time and $100 \%$ of slow wave sleep.

Mouth leak could lead to severe sleep fragmentation, either directly via airway irritation, or indirectly via a reduction in effectiveness of ventilation as discussed above. In the study by $\mathrm{BACH}$ et al. [7], there were a total of 34 arousals $\cdot \mathrm{h}^{-1}$ and $74 \%$ of desaturations were terminated by either an arousal or a lightening of sleep stage. The authors concluded that leak was reducing ventilatory effectiveness and causing desaturation, and that arousal reversed the leak and desaturation. Similarly, in the study of MEYER et al. [8] there were 46 arousals $\cdot \mathrm{h}^{-1}$ in stages I-II nonrapid eye movement (NREM) sleep, associated in time with mouth leak. However, association in time does not establish causality, and it is not known whether preventing the mouth leak would improve sleep quality.

The purpose of the present paper was to directly assess the effect of mouth leak on sleep architecture and transcutaneous carbon dioxide tension $\left(P \mathrm{tc}, \mathrm{CO}_{2}\right)$ during nasal bilevel ventilatory support, by preventing the leak. To do this, the authors measured the first-night effect of taping the mouth closed, on sleep architecture and $P \mathrm{tc}, \mathrm{CO}_{2}$ in patients already on long-term nasal bilevel ventilatory support and known to have mouth leak. In order to further explore to what extent the observed change in sleep and $P \mathrm{tc}, \mathrm{CO}_{2}$ might be explained by reduced ventilatory effectiveness, the effect of increasing mouth leak on ventilatorinduced hypopharyngeal pressure swings was measured 
directly in four awake healthy volunteers, and an approximate mathematical model constructed to estimate whether the order of magnitude of the observed changes was reasonable.

\section{Methods}

Ventilatory assistance during sleep: effect of taping the mouth

Nine patients were recruited from the outpatients department at a major chest hospital (in Germany) at the follow-up clinic for long-term home nasal bilevel ventilatory assistance for respiratory failure during sleep. Patients had presented originally with respiratory failure, hypoxia, or in two cases, cardiac failure and Cheyne-Stokes breathing. Patients were recruited if they had both complained of discomfort due to mouth leak, and had been previously observed to have significant mouth leak during ventilatory assistance in the laboratory. Patients had been on bilevel therapy for 2-11 months. Four patients were receiving supplemental oxygen therapy. All patients were being treated in spontaneous plus timed (ST) mode. Patient characteristics and ventilator settings are shown in table 1 . The original bilevel pressure settings were titrated over several days, to produce reasonable oxygen saturation, $P \mathrm{tc}, \mathrm{CO}_{2}$, and morning blood gases. Only respiratory monitoring, not polysomnography, was performed at the original titration in most patients. Since sleepiness was not the presenting symptom, original sleep architecture is unavailable. The protocol was approved by the Ethics Committee of the University of Essen, and patients gave written informed consent.

After recruitment, patients slept in the laboratory with bilevel nasal ventilatory assistance during polysomnography on two nights in random order. Ventilatory assistance was provided, and mask flow, pressure, and leak measured, using a specially modified AutoSet Clinical nasal CPAP system (ResMed, Sydney, Australia). The control software was modified to provide bilevel spontaneous plus timed mode ventilatory support.
The AutoSet Clinical system (ResMed) has a pneumotachograph placed between the exhaust port and the mask. The exhaust port is upstream of the pneumotachograph, and so the pneumotachograph is measuring true mask flow, independently of exhaust port flow. The software provides for calibrating the pneumotachograph with a known flow, and thence integrating flow to give tidal volume. This yields a tidal volume signal with a stated accuracy of $20 \%$. This study confirmed that the tidal volume (and therefore flow) was accurate to $10 \%$ using a $500 \mathrm{~mL}$ calibration syringe at 15 breaths $\cdot \min ^{-1}$.

Average leak was quantified by making use of the fact that over a sufficiently long period of time, the volume inspired by the patient approximately equals the volume expired. Therefore, in the absence of leak, the mean flow into the mask is close to zero. Conversely, if there is a leak of $1 \mathrm{~L} \cdot \mathrm{s}^{-1}$ then the mean flow into the mask (and out through the leak) will be $1 \mathrm{~L} \cdot \mathrm{s}^{-1}$. In general, the leak equals the mean flow into the mask. The AutoSet software calculates the leak by digitally low pass filtering the mask flow signal with a time constant of $10 \mathrm{~s}$. This time constant was chosen as being sufficiently long compared with a normal breath to filter out most of the respiratory airflow. For recording on the polygraph, the calculations were repeated in analogue hardware, by passing the analogue flow signal provided by the AutoSet blower through an analogue filter with a time constant of $10 \mathrm{~s}$ to give an analogue leak signal. This polygraph leak signal was then calibrated to agree with the software digital display. With the awake patient wearing a tightly fitting mask, mouth closed, reported leak was typically $0.0 \pm 0.05 \mathrm{~L} \cdot \mathrm{s}^{-1}$.

The flow generator servo-controlled the directly measured mask pressure to be substantially independent of leak up to a leak of $1.5 \mathrm{~L} \cdot \mathrm{s}^{-1}$. In this way, it was possible to assess the effect of mouth leak without the confounding influence of a decrease in mask pressure swings due to mask or mouth leak.

Pressure settings, backup respiratory rate, and supplemental oxygen flow rate were set as per the patient's long-term home therapy. A heated humidifier (Fisher and

Table 1. - Patient characteristics

\begin{tabular}{|c|c|c|c|c|c|c|c|c|c|c|c|c|}
\hline \multirow[t]{2}{*}{$\begin{array}{l}\mathrm{Pt} \\
\mathrm{n}\end{array}$} & \multirow[t]{2}{*}{ Primary diagnosis } & \multirow[t]{2}{*}{ Sex } & \multirow[t]{2}{*}{$\begin{array}{l}\text { Age } \\
\text { yrs }\end{array}$} & \multirow[t]{2}{*}{$\begin{array}{l}\text { AHI } \\
h^{-1}\end{array}$} & \multirow[t]{2}{*}{$\underset{\mathrm{kg} \cdot \mathrm{m}^{-2}}{\mathrm{BMI}}$} & \multirow[t]{2}{*}{$\begin{array}{l}\text { FEV } 1 \\
\% \text { pred }\end{array}$} & \multirow[t]{2}{*}{$\begin{array}{l}\mathrm{VC} \\
\% \text { pred }\end{array}$} & \multirow[t]{2}{*}{$\underset{\%}{\mathrm{FEV}_{1} / \mathrm{VC}}$} & \multicolumn{2}{|c|}{$P \mathrm{a}, \mathrm{CO}_{2} / \mathrm{Pa}_{\mathrm{a}} \mathrm{O}_{2} \mathrm{mmHg}$} & \multirow[t]{2}{*}{$\begin{array}{l}\text { IPAP/EPAP } \\
\mathrm{cmH}_{2} \mathrm{O}\end{array}$} & \multirow[t]{2}{*}{$\begin{array}{l}\text { Rate } \\
\min ^{-1}\end{array}$} \\
\hline & & & & & & & & & $\begin{array}{c}\text { On } \\
\text { admission }\end{array}$ & $\begin{array}{l}\text { On treatment } \\
\text { morning }\end{array}$ & & \\
\hline 1 & Kyphoscoliosis, COPD & $\mathrm{F}$ & 59 & 3 & 22 & 26 & 27 & 52 & $75 / 47$ & $53 / 69$ & $20 / 5$ & 24 \\
\hline 2 & $\begin{array}{l}\text { Central sleep apnoea due } \\
\text { to heart failure }\end{array}$ & M & 61 & 42 & 24 & 70 & 79 & 82 & $33 / 79$ & $35 / 81$ & $15 / 5$ & 18 \\
\hline 3 & $\begin{array}{l}\text { Central sleep apnoea due } \\
\text { to heart failure }\end{array}$ & M & 65 & 38 & 19 & 69 & 75 & 74 & $32 / 82$ & $37 / 78$ & $16 / 6$ & 18 \\
\hline 4 & Kyphoscoliosis, COPD & M & 48 & 6 & 26 & 21 & 38 & 39 & $68 / 45$ & $49 / 79$ & $18 / 5$ & 21 \\
\hline 5 & COPD & M & 67 & 5 & 27 & 22 & 36 & 42 & $62 / 55$ & $51 / 82$ & $15 / 7$ & 20 \\
\hline 6 & COPD, mild obesity & M & 72 & 7 & 29 & 26 & 42 & 53 & $68 / 48$ & $50 / 68$ & $19 / 5$ & 20 \\
\hline 7 & COPD & M & 74 & 3 & 19 & 22 & 38 & 39 & $73 / 49$ & $47 / 69$ & $15 / 6$ & 18 \\
\hline 8 & COPD & M & 59 & 4 & 20 & 20 & 38 & 35 & $69 / 56$ & $50 / 59$ & $19 / 7$ & 18 \\
\hline 9 & $\begin{array}{l}\text { COPD, mild obstructive } \\
\text { sleep apnoea, mild obesity }\end{array}$ & $\mathrm{M}$ & 69 & 11 & 31 & 24 & 32 & 48 & $71 / 52$ & $55 / 61$ & $20 / 6$ & 20 \\
\hline \multicolumn{2}{|c|}{ Mean } & & 64 & 13 & 24 & 33 & 45 & 52 & $61 / 57$ & $47 / 72$ & $17 / 6$ & 20 \\
\hline
\end{tabular}

Pt.: patient; AHI: apnoea-plus-hypopnoea index; BMI: body mass index; FEV1: forced expiratory volume in one second; VC: vital capacity; $\mathrm{Pa}_{\mathrm{a}} \mathrm{CO}_{2}$ : carbon dioxide tension in arterial blood; $\mathrm{Pa}, \mathrm{O}_{2}$ : oxygen tension in arterial blood; IPAP: inspiratory positive airway pressure; EPAP: expiratory positive airway positive; COPD: chronic obstructive pulmonary disease; F: female; M: male. 1 $\mathrm{mmHg}=0.133 \mathrm{kPa}$. 
Paykel, Auckland, New Zealand) was used in all cases. Sleep staging (2 electro-oculogram (EOG), 2 electroencephalogram (EEG), submental elctromyogram (EMG), and $P_{t c}, \mathrm{CO}_{2}$ (Radiometer TCM3, Copenhagen, Denmark) were measured using a digital polygraph (CompuMedics, Melbourne, Australia). Mask pressure and leak were recorded on the polygraph. Pulse oximetry and electrocardiography (ECG), with suitable alarms, and ribcage and abdominal movement were also recorded for safety.

On the control night, patients slept as usual. On the treatment night, conditions were identical to the control night excepting that the mouth was taped closed several minutes prior to lights out, using several layers of hypoallergenic medical grade adhesive tape. The last $5 \mathrm{~cm}$ of the tape was folded over to provide a nonadhesive quickrelease tab, and the patient shown how to use this. On both nights, great care was taken with mask fitting and headstrap adjustment, to minimize mask leaks.

The polygraph records were scored manually for sleep latency, sleep stage, and arousals. An arousal was defined as three contiguous seconds of alpha with concomitant increase in submental EMG. $P \mathrm{tc}, \mathrm{CO}_{2}$ was recorded at 0.5 $\mathrm{Hz}$, and mean overnight $P \mathrm{tc}, \mathrm{CO}_{2}$ calculated. Results for the control and taped nights were compared using paired ttests. Mask leak was similarly recorded, and 25th, 50th, and 75 th centiles calculated.

\section{Effect of mouth leak in awake control subjects}

Four control adults were studied. A catheter tip pressure transducer (Gaeltec Catheters, Rehlingen-Siersburg, Germany) was placed in the oropharynx. In 3 subjects, the catheter was initially introduced after topical anaesthesia. However, this caused considerable nasal congestion, so measurements were repeated on another occasion without local anaesthesia. In these awake experiments, nasal ventilatory support was using either a bilevel positive airway pressure ventilator in spontaneous plus timed mode, using either a BiPAP-ST (Respironics, Pittsburgh, USA) or a VPAP-ST (ResMed) set to an inspiratory/expiratory pressure of $25 / 10 \mathrm{cmH}_{2} \mathrm{O}$ at 15 breaths. $\mathrm{min}^{-1}$. In both cases, the nose mask and pneumotachograph from the AutoSet nasal CPAP system was used, to provide mask pressure and flow signals. As with the measurements during sleep, heated humidification was used in all experiments.

During nasal ventilation, the subject was asked to make no spontaneous respiratory efforts. Subjects were asked to produce graded leaks through the mouth, by closing the teeth, an regulating the escaping air using the lips. Mask pressure, leak, mask flow, and hypopharyngeal pressure were recorded on a digital polygraph (CompuMedics) at $125 \mathrm{~Hz}$. For periods of $\sim 60 \mathrm{~s}$ in which the leak was steady, the mean inspiratory and expiratory mask pressure and hypopharyngeal pressure were calculated, and the ratio of hypopharyngeal pressure swing to mask pressure swing calculated and plotted as a function of mouth leak.

\section{Mathematical model}

The combined nasal and palatopharyngeal resistance was modelled as a single orifice resistance (pressure drop proportional to the square of flow), with a pressure drop at a flow of $1 \mathrm{~L} \cdot \mathrm{s}^{-1}(\mathrm{KN})$ of $4 \mathrm{cmH}_{2} \mathrm{O}$ to represent a normal nasal resistance [9], or of 8 and $30 \mathrm{cmH}_{2} \mathrm{O}$ to represent a congested nasal mucosa, and a severely congested mucosa after prolonged mouth leak [9] respectively. The authors modelled the leak at the mouth as a second orifice resistance, which was adjusted to give a variety of leaks, and the glottis, lower airways, and lungs as a laminar resistance (pressure drop linear on flow) of $8 \mathrm{cmH}_{2} \mathrm{O}$ (to simulate the lower airway resistance in moderate obstructive lung disease) in series with a bellows of compliance $0.125 \mathrm{cmH}_{2} \mathrm{O} \cdot \mathrm{L}^{-1} \cdot \mathrm{s}^{-1}$ representing lung plus chest wall. The authors then numerically simulated the effect of bilevel ventilation in a centrally apnoeic subject with inspiratory positive airway pressure (IPAP)/ expiratory positive airway pressure (EPAP) $17 / 6 \mathrm{cmH}_{2} \mathrm{O}$ and 10 breaths $\mathrm{min}^{-1}$. (Equations are given in the Appendix). The model ignores flow limitation due to pharyngeal collapse and glottic narrowing with overventilation.

\section{Results}

\section{Mouth leak in ventilated patients during sleep}

The effect of mouth leak on mean overnight $P \mathrm{tc}, \mathrm{CO}_{2}$ is shown in table 2 and figure 1 . On the untaped (control) night, the total mask plus mouth leak was high (mean 0.4 $\mathrm{L} \cdot \mathrm{S}^{-1}$ ) and for $25 \%$ of the study, exceeding on average $0.61 \mathrm{~L} \cdot \mathrm{s}^{-1}$. There was negligible change in IPAP or EPAP over the range of leak encountered. On low-light video, the lips and cheeks could be seen to vibrate during times of high leak. Typically, the leak was through a small gap between the lips of the order of 1-4 mm height and 5-10 $\mathrm{mm}$ width, typically at the angle of the mouth, the upper and lower lip being in contact over most of their length. On the taped night, leak was largely abolished in all but one patient, who still had a persistent mask leak. Mean overnight $\mathrm{Ptc}_{\mathrm{tc}} \mathrm{CO}_{2}$ fell often substantially in all but one patient (who was initially hypocapnic).

\section{Mouth leak and sleep architecture}

Patients tolerated the mouth tape surprisingly well, and the tape was still securely in place in the morning, patients reporting that the seal was still intact. The effect of taping the mouth on sleep architecture is shown in table 3 and figure 2 . All nine patients showed a reduction in arousal index with taping of the mouth, and on average the arousal index was more than halved with taping. There was an increase in REM sleep in all but one patient, and on average the percentage of REM sleep increased by $>50 \%$. There were inconsistent changes in slow wave sleep. There were no important differences in study duration,

Table 2. - Effect of taping mouth on total (mouth plus mask) leak

\begin{tabular}{lcccl}
\hline & Control & Taped & Delta & p-value \\
\hline Leak L $\cdot \mathrm{s}^{-1}$ & & & & \\
25th centile & $0.08 \pm 0.01$ & $0.03 \pm 0.02$ & $-0.05 \pm 0.02$ & 0.05 \\
Median & $0.35 \pm 0.07$ & $0.06 \pm 0.03$ & $-0.29 \pm 0.08$ & 0.008 \\
75th centile & $0.61 \pm 0.08$ & $0.09 \pm 0.04$ & $-0.52 \pm 0.09$ & 0.0005 \\
\hline
\end{tabular}

Data are presented as mean \pm SEM. 


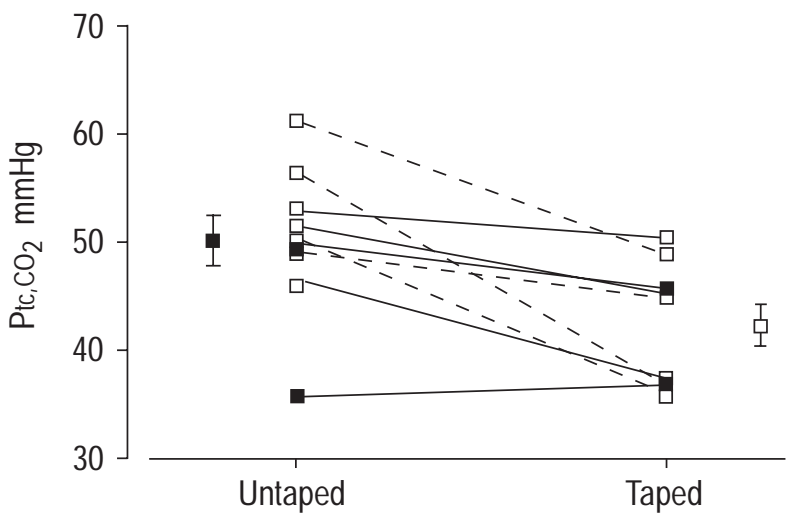

Fig. 1. - Mean overnight transcutaneous carbon dioxide tension $\left(P \mathrm{tc}, \mathrm{CO}_{2}\right)$ during bilevel ventilatory assistance. $\square$ : cardiac failure with Cheyne Stokes breathing; $\square$ : chronic obstructive pulmonary disease $(\mathrm{n}=6)$ or kyphoscoliosis $(\mathrm{n}=1)$; - - patients receiving supplemental oxygen on both nights $(n=4) ;---$ : patients not receiving supplemental oxygen $(\mathrm{n}=5) ;|-\mathbf{\square}-|$ : mean \pm SEM for untaped mouth leakage; $|-\square-|$ : mean \pm SEM for taped mouth leakage.

sleep efficiency, or sleep latency between the taped and untaped nights. Although numbers were small, there were no obvious differences in the effect of taping the mouth on sleep architecture between the patients with and without supplemental oxygen, or between the eucapnic and hypercapnic patients.

\section{Mouth leak and hypopharyngeal pressure in control subjects}

Figure 3 shows mask pressure, mask airflow and leak, and hypopharyngeal pressure plotted against time in a control subject. There was a small fall in mask pressure swings with leak in these experiments, because (unlike the studies during sleep) the mask pressure was not directly servo-controlled, and the blower was not designed to compensate for the pressure drop introduced by the pneumotachograph. As expected, there was a large decrease in both inspiratory and expiratory hypopharyngeal pressure. Figure 4 shows the ratio of hypopharyngeal pressure swings to mask pressure swings as a function of leak for each of the four subjects, along with the calculated $K N\left(\right.$ in $\mathrm{cmH}_{2} \mathrm{O} \cdot \mathrm{L}^{-2} \cdot \mathrm{s}^{-2}$ ) for each test run. The dotted lines show the tests performed after local anaesthesia, where $K N$ is greatly increased due to nasal congestion. In the four tests without local anaesthesia, the mean KN was

Table 3. - Effect of taping mouth on sleep architecture

\begin{tabular}{|c|c|c|c|c|}
\hline & Control & Taped & Delta & p-value \\
\hline $\begin{array}{l}\text { Time available for } \\
\text { sleep min }\end{array}$ & $372 \pm 12$ & $390 \pm 9$ & $18 \pm 15$ & 0.3 \\
\hline Total sleep time min & $309 \pm 13$ & $341 \pm 18$ & $32 \pm 23$ & 0.2 \\
\hline $\begin{array}{l}\text { Sleep efficiency \% } \\
\text { time available }\end{array}$ & $83.1 \pm 2.7$ & $86.9 \pm 3.2$ & $3.8 \pm 3.8$ & 0.3 \\
\hline Sleep latency $\min$ & $14.2 \pm 1.8$ & $12.3 \pm 2.1$ & $-1.9 \pm 2.3$ & 0.4 \\
\hline $\begin{array}{l}\text { Slow wave sleep \% } \\
\text { sleep time }\end{array}$ & $13.1 \pm 1.6$ & $19.5 \pm 2.2$ & $6.4 \pm 3.4$ & 0.09 \\
\hline $\begin{array}{l}\text { REM sleep \% sleep } \\
\text { time }\end{array}$ & $12.9 \pm 1.5$ & $21.1 \pm 1.8$ & $8.1 \pm 1.8$ & 0.0016 \\
\hline
\end{tabular}

Data are presented as mean \pm SEM. REM: rapid eye movement.

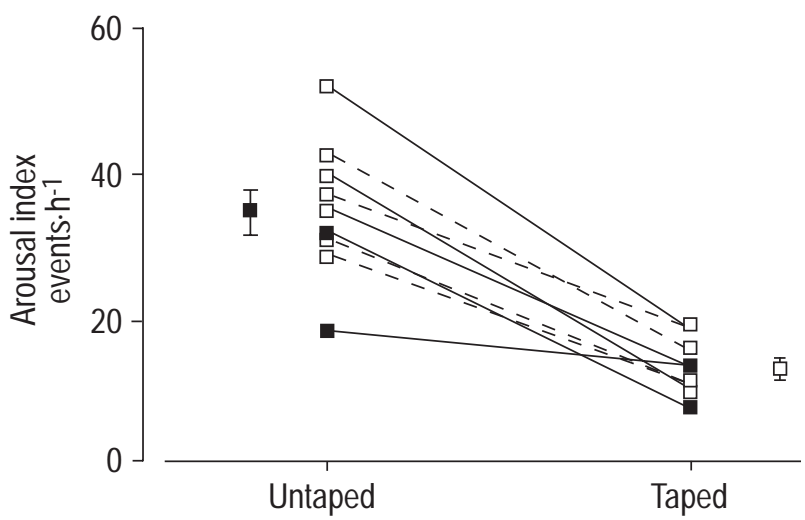

Fig. 2. - Effect of taping the mouth on arousal index. $\square$ : cardiac failure with Cheyne Stokes breathing; $\square$ : chronic obstructive pulmonary disease $(\mathrm{n}=6)$ or kyphoscoliosis $(\mathrm{n}=1)$; - : patients receiving supplemental oxygen on both nights $(\mathrm{n}=4) ;-{ }_{-}-$: patients not receiving supplemental oxygen $(\mathrm{n}=5) ;|-\mathbf{-}-|:$ mean \pm SEM for untaped mouth; $|-\square-|$ : mean \pm SEM for taped mouth

$5.5 \mathrm{cmH}_{2} \mathrm{O} \cdot \mathrm{L}^{-2} \cdot \mathrm{s}^{-2}$, and the ratio of hypopharyngeal to mask pressure swings decreased by $35 \pm 5 \%$ per $\mathrm{L} \cdot \mathrm{s}^{-1}$ of mouth leak $(\mathrm{p}<0.0001)$. In the three tests with local anaesthesia $K N$ was $20 \mathrm{cmH} \mathrm{O}_{2} \mathrm{O} \cdot \mathrm{L}^{-2} \cdot \mathrm{s}^{-2}$, and the ratio of hypopharyngeal to mask pressure swings was low (mean 0.4 ) even with the mouth closed, decreasing by a further $36 \pm 12 \%$ per $L \cdot s^{-1}$ of mouth leak $(p=0.017)$.

\section{Mathematical model}

Simulated hypopharyngeal pressure was markedly reduced by mouth leak, during both ventilator inspiration and expiration, and the amplitude of the excursions in hypopharyngeal pressure were greatly reduced. The ratio of the pressure swing at the hypopharynx to the pressure swing at the mask is plotted in figure 5 for a control and a congested $\mathrm{KN}$.

\section{Discussion}

This is the first detailed study of the effect of preventing mouth leak on either sleep architecture or $P \mathrm{tc}, \mathrm{CO}_{2}$ during nasal bilevel ventilatory assistance in sleep. This study clearly demonstrated that when patients are receiving noninvasive ventilatory support via the nasal airway during sleep, the occurrence of mouth leak reduces effective ventilation, induces multiple arousals, and reduces the amount of REM sleep: the prevention of mouth leaks improved ventilation, greatly reduced arousals, and markedly increased REM sleep. It is speculated that these physiological improvements will lead to improved clinical outcome, and, therefore, preventing mouth leaks is an important goal when attempting to achieve fully effective nasal ventilatory support during sleep.

\section{Effectiveness of ventilatory support}

On average, patients showed a worthwhile reduction in $P \mathrm{tc}, \mathrm{CO}_{2}$ with the mouth taped closed, implying an improvement in effectiveness of ventilatory support. The first possible mechanism is an improvement in hypopharyngeal 


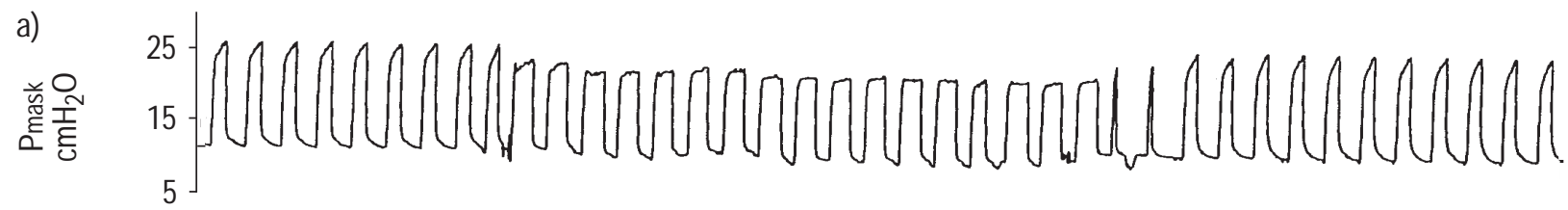

b)


d)

Time $\min$

Fig. 3. - Effect of voluntary awake mouth leak of $0.5-0.7 \mathrm{~L} \cdot \mathrm{s}^{-1}$ on hypopharyngeal pressure in a typical awake control subject. Subject is making no respiratory efforts. a) mask pressure (Pmasks), b) leak, c) mask flow, and d) hypopharyngeal pressure (Phyp), plotted against time. Width of trace: 3.3 min.

pressure swing for a given mask pressure swing. It was decided not to measure the hypopharyngeal pressure swings directly during sleep in this study, because the presence of the catheter would have greatly complicated the interpretation of changes in sleep architecture, as well as introducing mask leaks and changes in nasal resistance. Therefore, the relationship between leak and ventilatory support in awake control subjects was measured, and also a mathematical simulation performed. Both approaches showed that increasing mouth leak caused a dose dependent

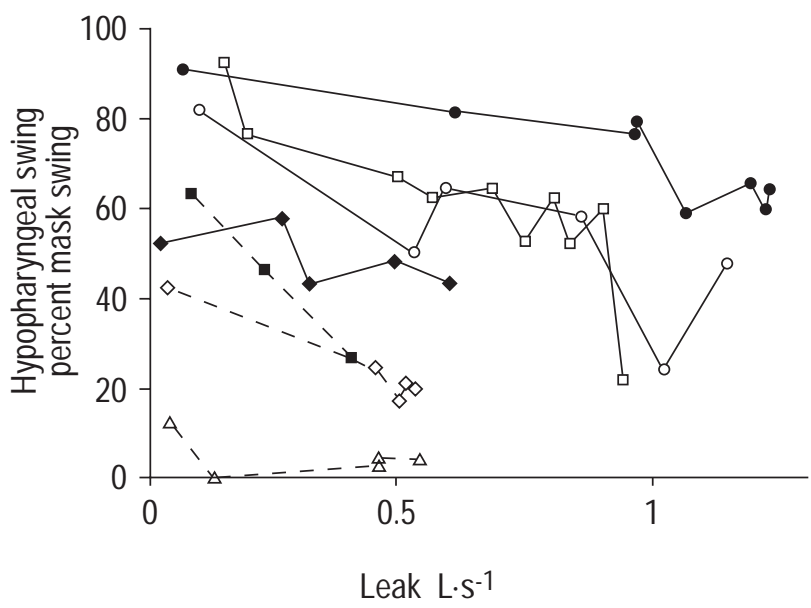

Fig. 4. - Effect of voluntary awake mouth leak on ratio of hypopharyngeal to mask pressure swing. — : hypopharyngeal catheter introduced without local anaesthesia $(n=4) ;---$ : after unintended nasal congestion due to use of topical anaesthesia $(n=3)$. The mean nasal resistance $\left(\mathrm{cmH}_{2} \mathrm{O} \cdot \mathrm{L}^{-2} \cdot \mathrm{s}^{-2}\right)$ for each of the seven tests were; $\bigcirc: 8.5 ; 0: 2.3 ; \square: 3.6$;

: $7.4 ; \diamond: 14.9 ; \diamond: 23.6 ; \Delta: 22.8$. reduction in hypopharyngeal pressure, and a reduction in hypopharyngeal pressure swings, which was quite modest for typical leaks and nasal resistances, but became very large for high leaks, particularly if there was nasal congestion. Part of the pressure drop in control subjects may have been due to flow limitation at very high flows, as suggested by the shape of the flow-time curve in figure 3 .

Nasal congestion will greatly accentuate the pressure drop with leak. The mean $K N$ in the four control subjects was $5.5 \mathrm{cmH}_{2} \mathrm{O} \cdot \mathrm{L}^{-2} \cdot \mathrm{s}^{-2}$, close to the value of $4.4 \mathrm{cmH}_{2} \mathrm{O}$. $\mathrm{L}^{-2} \cdot \mathrm{s}^{-2}$, (reported as a linear resistance of $1.1 \mathrm{cmH}_{2} \mathrm{O} \cdot \mathrm{L}^{-2}$. $\mathrm{s}^{-2}$ at $\left.0.5 \mathrm{~L} \cdot \mathrm{s}^{-1}\right)$ of RichARDs et al. [9] in six healthy volunteers. HAYES et al. [10] showed that mouth leak

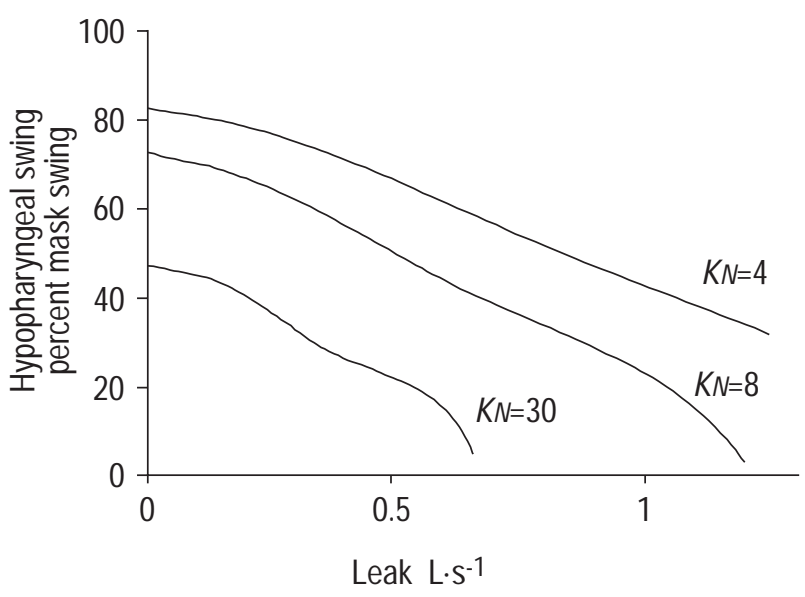

Fig. 5. - Mathematical model. Predicted effect of mouth leak on the ratio of hypopharyngeal to mask pressure swing, for a pressure drop at 1 $\mathrm{L} \cdot \mathrm{s}^{-1}(K N)$ of 4,8 , and $30 \mathrm{cmH}_{2} \mathrm{O}$. 
caused a great increase in nasal mucosal bloodflow and a reduction in lumen, largely preventable by humidification. Richards et al. [9] showed that in the absence of humidification, mouth leak $K N$ rose to $30 \mathrm{cmH}_{2} \mathrm{O} \cdot \mathrm{L}^{-2} \cdot \mathrm{s}^{-2}$, and that this effect was greatly reduced (but not eliminated) by humidification. Consequently, it might be speculated that in the absence of a heated humidifier, the effect of mouth leak on effectiveness of ventilation might be more severe.

It is not possible to calculate whether the predicted improvement in the degree of ventilatory support with taping fully accounts for the observed $1.02 \mathrm{kPa}(7.7 \mathrm{mmHg})$ decrease in $P \mathrm{tc}, \mathrm{CO}_{2}$ with taping because neither the $\mathrm{KN}$ for these particular sleeping patients, nor the relationship between support and $P \mathrm{tc}, \mathrm{CO}_{2}$ is known.

The second possible mechanism for the observed 1.02 $\mathrm{kPa}(7.7 \mathrm{mmHg})$ decrease in $P \mathrm{tc}, \mathrm{CO}_{2}$ without taping is due to worse gas exchange due to loss of expiratory pressure, as shown for patients with neuromuscular/skeletal disorders by ElliotT and Simmonds [11]. A third possible mechanism is that mouth leak may cause mistriggering of the ventilator. Fourthly, a reduction in hypopharyngeal pressure with mouth leak may unmask obstructive sleep apnoea.

The improvement is despite an increase in REM sleep, which of itself is a time of increasing $\mathrm{PCO}_{2}$ in subjects with hypercapnic respiratory failure. Conversely, the initially hypocapnic patient with central sleep apnoea due to cardiac failure in the current study did not show a further reduction in $P \mathrm{tc}, \mathrm{CO}_{2}$. This could simply reflect regression toward the mean. Equally, it might be speculated that unlike the patients with lung or chest wall disease, this patient with normal lungs was able to spontaneously maintain mean alveolar ventilation in the face of decreased ventilator support.

The change in ventilatory support with leak was not due to a change in mask pressure. For the studies during sleep, the mask pressure was servo-controlled to be independent of mask leak over the range of leaks encountered in the sleeping patients. In the control subjects, changes in mask pressure (and any direct current (DC) transducer drift) were compensated for by expressing hypopharyngeal pressure swings as a percentage of mask pressure swings.

\section{Vocal cord closure}

PARRIERA and coworkers $[12,13]$ have shown in control subjects that excessive ventilatory support with a volume cycled ventilator, or with a bilevel ventilator in the timed (but not spontaneous) mode causes reflex glottic narrowing. Firstly, this will greatly reduce the degree of ventilatory support. Secondly, with a volume cycled ventilator, this will lead to an enormous increase in airway pressure and cause mouth leak. Therefore, excess support should be considered as a cause of mouth leak, particularly with volume cycled machines.

\section{Sleep architecture}

The most striking result of the present study was that taping the mouth for one night halved the arousal index, and produced a large increase in the percentage of REM sleep, with no deterioration in the sleep latency or sleep efficiency. This was a very large and clinically significant effect. It is as yet uncertain how much of this effect can be maintained long-term. However, the improvements are of the same order of magnitude as seen in the treatment of obstructive sleep apnoea with nasal CPAP, and may well translate to an improvement in quality of life.

Perhaps the most crucial result of the study in the awake control subjects was that all four control subjects reported extreme discomfort with large mouth leaks, even with a heated humidifier. During sleep, large amplitude vibration of the lips and cheeks with mouth leak were observed. Since patients are asleep during mouth leak, they may be unaware of the cause of their arousals. It is therefore speculated that relief of leak-related discomfort may be the main cause of the improvement in sleep architecture with prevention of mouth leak. The second main mechanism which may be leading to an improvement in sleep quality is an improvement in ventilatory support. There was, on average, a $1.02 \mathrm{kPa}(7.7 \mathrm{mmHg})$ reduction in $P \mathrm{tc}, \mathrm{CO}_{2}$, and this would cause a large change in chemoreceptor drive in some patients.

Some other possible mechanisms cannot be excluded: 1) the first is prevention of loss of supplemental oxygen via the mouth. Again, such an effect might be extremely important in some patients, particularly severely hypoxic patients with intact ventilatory responses to hypoxia. In the present study, four patients received supplemental oxygen, and five (including the two with central sleep apnoea) did not. However, all nine patients showed an improvement in arousal index, so oral loss of supplemental oxygen cannot be the only mechanism for improved arousal index; 2) there may be a placebo effect. Patients were aware of the purpose of the study, and it was not possible to blind them to the presence or absence of the tape. However, it seems implausible that taping the mouth in an anxious and dyspnoeic patient would of itself improve sleep; 3) it is not possible to exclude a fortuitous reduction in mask leak with taping of the mouth, but this is most unlikely; 4) one patient had coexisting obstructive sleep apnoea (apnoeaplus-hypopnoea index $11 \cdot \mathrm{h}^{-1}$ ), and a reduction in hypopharyngeal splinting could explain some or all of the increased arousal in this patient.

There is an urgent clinical need for a safe and effective method for preventing mouth leaks during bilevel ventilatory support. Taping was used in this study in preference to other ways of sealing the mouth such as a full face mask, because existing full face masks have higher deadspace, leak, and discomfort than nosemasks, and arousal data would be uninterpretable. However, the authors do not at this stage advocate taping the mouth for indiscriminate long-term home use, because of the risk of asphyxia in the presence of nasal obstruction, machine or power failure, or regurgitation.

Since performing the present study, $\sim 30$ carefully chosen patients, relying on nocturnal support for ventilation but unable to be satisfactorily treated due to mouth leak, unable to tolerate a full face mask, highly alert, with no history of regurgitation, and faced with the alternatives of tracheotomy or untreated respiratory insufficiency, have elected to use tape at home for periods of up to 6 months. The tape is well tolerated and no adverse events have been observed. In one patient, taping the mouth caused the $\mathrm{Ptc}_{\mathrm{tc}} \mathrm{CO}_{2}$ to fall acutely from $8.91 \mathrm{kPa}$ to $5.32 \mathrm{kPa}(67$ $\mathrm{mmHg}$ to $40 \mathrm{mmHg}$ ), and it was necessary to reduce the ventilator settings to compensate. 
This study was confined to known symptomatic mouth leakers. However, $\sim 100 \%$ of the population of noninvasively ventilated patients would appear to have clinically relevant mouth leak $[7,8]$, which should be routinely measured, particularly if the nasal resistance is high, if treatment is not fully successful, or if sleep architecture appears to be impaired.

In conclusion, during bilevel ventilatory support, severe mouth leak can cause an important reduction in the ventilator-induced pressure swings at the hypopharynx, particularly if the nose is congested. High mouth leak is very distressing to awake control subjects and is a likely cause of severe sleep disruption. On the first night, taping the mouth closed led to more than halving of the arousal index and a worthwhile increase in rapid eye movement sleep, with no increase in sleep latency or reduction in sleep efficiency, and led to a reduction in transcutaneous carbon dioxide tension in hypercapnic patients. There is a need for routine assessment of the degree of mouth leak in nasally ventilated patients, with a view to preventing mouth leak where possible.

\section{Appendix: Mathematical model}

The chiefly turbulent flow pressure drop from mask to hypopharynx was taken as $P M-P H=K N f N^{2}$ where $P M$ is mask pressure, $P H$ is hypopharynx pressure, $f_{N}$ is the nasal airflow and $K N$ is a constant. Similarly the pressure drop from hypopharynx to room (via the mouth) was taken as $P H=K M f \mathrm{M}^{2}$ where $f M$ is the instantaneous leak through the mouth and $K M$ is a constant. The chiefly laminar flow pressure drop from pharynx to alveoli was taken as $\mathrm{PH}^{-}$ $P A=R L f L$ where $P A$ is alveoli pressure, $R L$ is the lower airway resistance and $f L$ is the flow into the lungs. Finally, the pressure in the alveoli $(P A)$ was taken as proportional to volume, whence the time rate of change $(d t)$ of alveolar pressure is given by $d P A / d t=f L / C$ where $C$ is the compliance of lung and chest wall.

\section{References}

1. Ellis EB, Bye PT, Bruderer JW, Sullivan CE. Treatment of respiratory failure during sleep in patients with neuromuscular disease: positive-pressure ventilation through a nose mask. Am Rev Respir Dis 1987; 135: 148-152.
2. Bach JR, Alba A, Mosher R, Delaubier A. Intermittent positive pressure ventilation via nasal access in the management of respiratory insufficiency. Chest 1987; 92: 168-170.

3. Ambrosino N, Nava S, Bertone PT, Fracchia C, Rampulla C. Physiologic evaluation of pressure support ventilation by nasal mask in patients with stable COPD. Chest 1992; 101: 385-391.

4. Pepin JL, Leger P, Veale D, Langevin B, Robert D, Levy P. Side effects of nasal continuous positive airway pressure in sleep apnea syndrome. Chest 1995; 107: 375-381.

5. Hoffstein V, Viner S, Mateika S, Conway J. Treatment of obstructive sleep apnea with nasal continuous positive airway pressure: patient compliance, perception of benefits and side effects. Am Rev Respir Dis 1992; 145: 841845 .

6. Carrey Z, Stewart G, Levy RD. Ventilatory muscle support in respiratory failure with nasal positive pressure ventilation. Chest 1990; 97: 150-159.

7. Bach J, Robert D, Leger P, Langevin B. Sleep fragmentation in kyphoscoliotic individuals with alveolar hypoventilation treated by NIPPV. Chest 1995; 107 : $1552-1558$.

8. Meyer TJ, Pressman MR, Benditt J, et al. Air leaking through the mouth during nocturnal nasal ventilation: effect on sleep quality. Sleep 1997; 20: 561-569.

9. Richards GN, Cistulli PA, Ungar G, Berthon-Jones M, Sullivan CE. Mouth leak with nasal continuous positive airway pressure increases nasal airway resistance. $\mathrm{Am} \mathrm{J}$ Respir Crit Care Med 1996; 154: 182-186.

10. Hayes MJ, McGregor FB, Roberts DN, Schroter RC, Pride NB. Continuous nasal positive airway pressure with a mouth leak: effect on nasal mucosal blood flux and nasal geometry. Thorax 1995; 50: 1179-1182.

11. Elliott MW, Simonds AK. Nocturnal assisted ventilation using bilevel positive airway pressure: the effect of expiratory positive airway pressure. Eur Respir $J$ 1995; 8: 436-440.

12. Parreira VF, Delguste P, Jounieaux V, Aubert G, Dury M, Rodenstein DO. Glottic aperture and effective minute ventilation during nasal two-level positive pressure ventilation in spontaneous mode. Am J Respir Crit Care Med 1996; 154: 1857-1863.

13. Parreira VF, Jounieaux V, Aubert G, Dury M, Delguste PE, Rodenstein DO. Nasal two-level positive-pressure ventilation in normal subjects. Effects of the glottis and ventilation. Am J Respir Crit Care Med 1996; 153: 16161623. 